\title{
Modelo de estados financieros en base a las Normas Internacionales de Información Financiera (NIIF)
}

\author{
Financial statement model based on international financial reporting standards. \\ Karla Isabel Malo Cruz ${ }^{a}$, Irma Isabel de León Vázquez, ${ }^{b}$, Ruth Flores Jiménez ${ }^{c}$
}

\begin{abstract}
:
This essay analyzes the importance of having financial instruments within organizations based on the International Financial Reporting Standards, considering as problematic that there are institutions that have archaic Financial Statements. The purpose is to help accounting globalization and universal understanding in financial information. Several concepts related to the subject were investigated and organizations are considered to be constantly updated regarding their financial models to achieve a clear and precise interpretation of their finances.
\end{abstract}

Keywords:

Financial instruments, IFRS, Financial Information, archaic

\section{Resumen:}

El presente ensayo analiza la importancia de contar con instrumentos financieros dentro de las organizaciones con base en las Normas Internacionales de Información Financiera, considerando como problemática que existe instituciones que cuentan con Estados Financieros arcaicos. La finalidad es ayudar a la globalización contable y al entendimiento universal en la información financiar. Se investigaron varios conceptos relacionados con el tema y se considera que las organizaciones deben actualizarse constantemente en cuanto a sus modelos financieros para lograr una clara y precisa interpretación de sus finanzas.

Palabras Clave:

Instrumentos financieros, NIIF, Información Financiera, arcaicos

\section{Introducción}

El presente ensayo se conforma por cinco apartados, el primero habla sobre la importancia de los estados financieros y cuales se consideran como principales; el segundo menciona los principales objetivos de los estados financieros; el tercero se refiere a la importancia de las Normas de Información financiera dentro de los estados financieros; el cuarto hace referencia a las Normas Internacionales de Información Financiera considerando los Estados Financieros y, por ultimo; analizamos las herramientas Financieras como apoyo para la toma de decisiones y mencionando algunos métodos de recopilación de datos para obtener información financiera concisa. Se muestra en cada uno de ellos un claro concepto y una concisa información obtenida de diversos recursos de información sobre el análisis de los Estados Financieros, donde la organización, los administradores y carreras afines se permitan conocer en qué situación económica se encuentra una empresa, y así mismo considerar posibles escenarios futuros. De esta manera se podrá dar un diagnóstico y brindar posibles sugerencias para alcanzar las metas trazadas dentro de cualquier organización.

\footnotetext{
a Autor de Correspondencia, Universidad Autónoma del Estado de Hidalgo, Escuela Superior de Tlahuelilpan, Alumno de la Licenciatura en Administración, Email: ma341270@uaeh.edu.mx

${ }^{\mathrm{b}}$ Universidad Autónoma del Estado de Hidalgo, Escuela Superior de Tlahuelilpan, Profesor por Asignatura de la Licenciatura en Administración, Email: irmalv@uaeh.edu.mx

b Universidad Autónoma del Estado de Hidalgo, Escuela Superior de Tlahuelilpan, Profesor Investigador de la Licenciatura en Administración, Email: ruth@uaeh.edu.mx
} 


\section{Desarrollo}

\section{IMPORTANCIA DE LOS ESTADOS FINANCIEROS}

Los estados financieros, son una parte esencial de la contabilidad interna de la organización, se considera de gran utilidad para que exista una correcta toma de decisiones y pueda funcionar de manera adecuada. Por ello es fundamental analizarlos, siendo los estados financieros de mayor importancia:
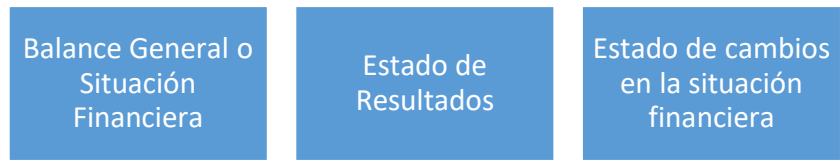

Estado de

variación al capital contable

Estado de costo de

producción y costo

de producción de

lo vendido
Parafraseando a Ochoa (2003, pág. 11), los Estados Financieros se definen de la siguiente manera:

\section{Balance general:}

Este estado financiero refleja la situación financiera que presenta una empresa en una fecha definida, por lo general se presenta de manera anual. Dicho documento se conforma por tres grandes rubros:

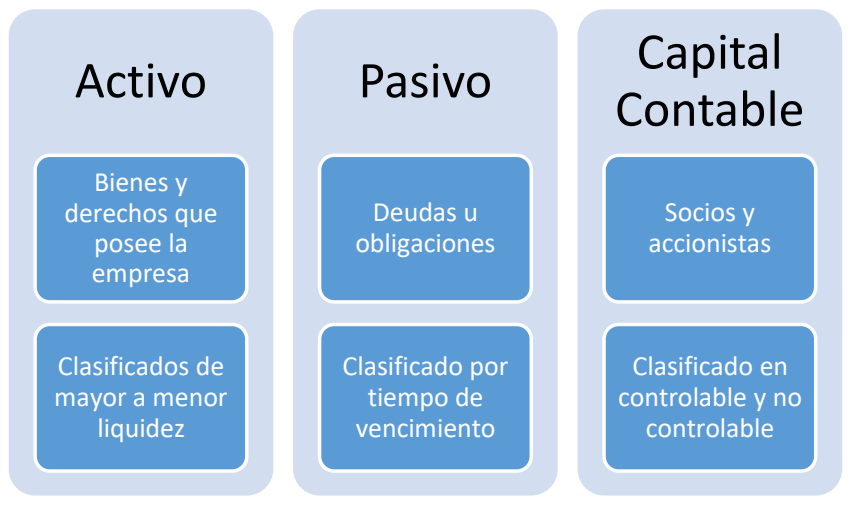

\section{Estado de resultados:}

También conocido como estado de ganancias o pérdidas, es un instrumento contable que muestra cómo se consiguieron los ingresos obtenidos, los costos de la compañía, los gastos en el momento en que se producen, y la forma en la que se obtuvo el beneficio o la pérdida neta generada a partir de las operaciones de la empresa durante un periodo determinado.

Considerando a Hurtado (2014, pág. 15), el estado de resultados ayuda a el gerente a conocer cuál ha sido el comportamiento mostrado por la empresa, si se han generado ganancias o pérdidas durante el periodo, con él puede estimar flujos de efectivo, capacidad de crédito, medir riegos, determinar si el proyecto es viable, etcétera.

La estructura de este Estado financiero se centraliza de la siguiente manera:

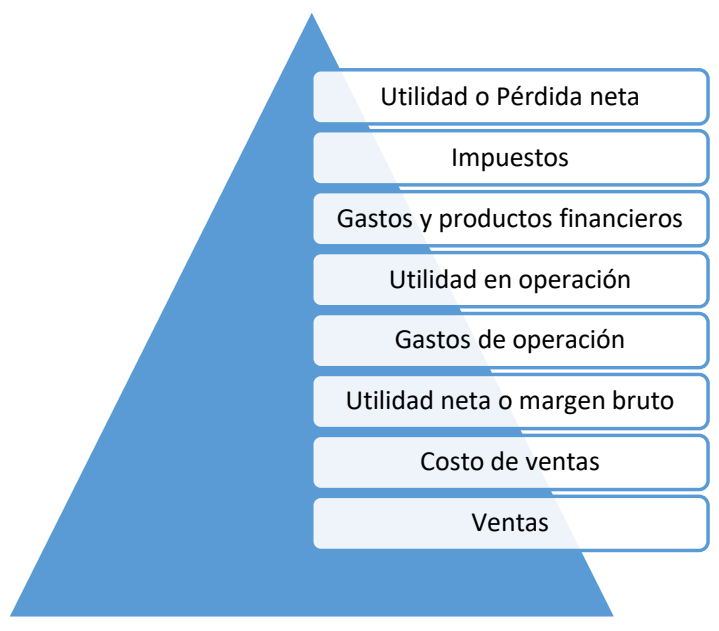

\section{Estado de cambios en la situación financiera:}

Estado financiero basado en el flujo de efectivo, muestra el origen de los recursos, y la forma en como los obtuvo, es decir, si fue de manera normal o a través de financiamientos, así mismo, ayuda en el análisis de las ganancias obtenidas, el monto disponible que se tiene en caja y bancos; señala dónde se ocupan los recursos, ya sea para ser invertidos en activos o los observe la misma operación normal de la empresa. De manera que el análisis de este instrumento financiero ayudará a evaluar los resultados y las decisiones gerenciales.

\section{Estado de variaciones en el capital contable:}

Según Ochoa (2003, pág. 11), documento contable que analiza los cambios en las cuentas de capital (capital contribuido y capital ganado). Este estado financiero es presentado por las organizaciones con fines lucrativos, ya que las no lucrativas no poseen un capital contable sino patrimonio.

Por otro lado, de acuerdo a (HURTADO ROMERO, 2014), éste muestra las variaciones de los diferentes elementos del patrimonio en un periodo determinado. 
Los movimientos de los propietarios se conforman de los siguientes conceptos.

- Aportaciones de capital (aumentos).

- Reembolso de capital (disminuciones).

- Decreto de dividendos (disminuciones).

- $\quad$ Capitalización de partidas de capital contribuido.

- Capitalización de utilidades o pérdidas integrales.

- Capitalización de reservas creadas.

\section{Estado de costo de producción y costo de producción de lo vendido:}

Es un estado financiero que se puede observar de manera más clara empresas industriales, pero también se utiliza en empresas comerciales y de servicio.

El costo de venta se conoce al producir o adquirir el producto que posteriormente será vendido, su principal objetivo es proporcionar información útil, confiable y oportuna. Se consideran cada uno de los elementos que participan en la elaboración de un producto, dicha información es utilizada en el estado de situación financiera (inventarios) y en el estado de resultados (costo de vetas).

El estado de costo de producción y de lo vendido considera: estado de costo de productos terminados (costos de materia prima, entre otros, relacionados con la producción) y estado de costo de lo vendido (costo de producción vendido).

\section{OBJETIVOS DE LOS ESTADOS FINANCIEROS}

Es importante conocer cuáles son los objetivos que se tienen al realizar los estados financieros.

"Los objetivos de los Estados Financieros resultan principalmente de las necesidades del usuario general, las cuales dependen significativamente de la naturaleza de las actividades de la entidad y de la relación que dicho usuario tenga con ésta" (Felipe, Allan, \& Antonio, 2019).

Los Estados Financieros deben permitir al usuario general evaluar lo mencionado en la Figura 1.

Los estados financieros deben ser útiles para tomar decisiones de inversión, saber si se puede otorgar créditos a proveedores y acreedores, evaluar los flujos de efectivo, evaluar la capacidad para tomar decisiones gerenciales, evaluar proyectos, conocer la capacidad de crecimiento de las empresas, entre otros.

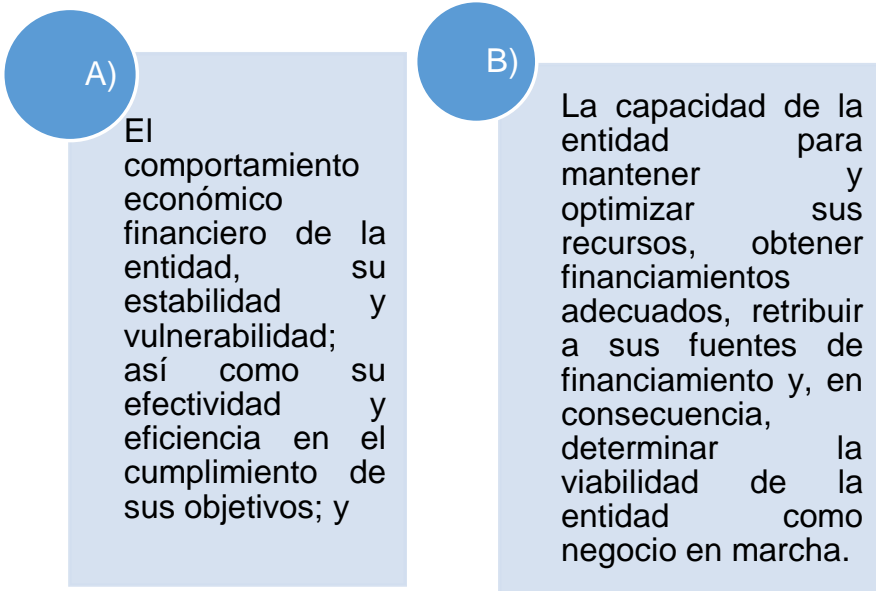

Figura 1. Fuente Propia obtenida de (Felipe, Allan, \& Antonio, 2019, pág.8)

\section{IMPORTANCIA DE LAS NORMAS DE INFORMACIÓN FIANCIERA DENTRO DE LOS ESTADOS FINANCIEROS}

La contabilidad ha contado con principios y ahora con normas que regulan la elaboración y presentación de la información financiera las NIF (Normas de Información Financiera) son las que actualmente se encuentran en función y son las que nos ayudan a establecer los registros y presentación de los estados financieros. Las NIF con el paso del tiempo reemplazaron a los Principios de Contabilidad.

Las Normas de Información Financiera son necesarias y forzosamente deben aplicarse a todas aquellas entidades que manejan información financiera.

Por otro lado también se buscó homologar al cambio normativo internacional dando la aceptación de las Normas Internacionales de Información Financiera que surge de un análisis realizado por la CINIF (Consejo Mexicano de Normas de Información Financiera A.C.).

\section{NORMAS INTERNACIONALES DE INFORMACIÓN FIANCIERA CONSIDERANDO LOS ESTADOS FINANCIEROS}

En varios países del mundo las Normas Internacionales de Información Financiera (NIIF), o International Financial 
Reporting Standards (IFRS- siglas en inglés) contienen nuevos estándares en reemplazo de los Principios de Contabilidad Generalmente Aceptados (PCGA), incluye cambios que modifican las bases de registro en la contabilidad.

Parafraseando a (Deloitte, 2019) se hace referencia a algunos cambios como: la medición inicial y posterior de los activos y pasivos a valor razonable vs. Costo histórico, moneda funcional vs. Moneda local, registro de la esencia económica vs. Forma legal, condiciones para dar de baja un activo financiero, en contratos de construcción se pueden mostrarse conforme ingresos y gastos de acuerdo al avance de la obra, los activos biológicos -ingresos, mayor valor por crecimiento y la estimación de sus costos de ventas-, corrección monetaria solo en economías hiperinflacionarias, reconocimiento del desperfecto del valor de los activos, información financiera por segmentos.

De acuerdo a los lineamientos establecidos dentro de las NIFF, queda prohibido el método LIFO (ultimo en entrar, primero en salir), en inventarios, en los estados financieros consolidados no hay exclusiones, activos intangibles no se amortizan sino que requieren prueba de deterioro, obligaciones con los empleados, registro de derivados implícitos, valoración de los instrumentos financieros por valor razonable o costo amortizado, diferenciación entre propiedad, planta y equipo con arrendamientos financieros y propiedades de inversión.

Estos aspectos se tratan en los lineamientos de Estados Financieros Básicos bajo NIC/NIIF dentro de sus particularidades

\section{HERRAMIENTAS FINANCIERAS COMO APOYO PARA LA TOMA DE DECISIONES}

Las herramientas financieras son un medio que nos ayudan a identificar el comportamiento de las empresas, son de gran utilidad ya que permiten simplificar los estados financieros, logrando observar escenarios futuros dentro de la organización. Estas herramientas financieras contribuyen a la recopilación de información, es decir, nos van a permitir tener un mejor control favoreciendo a la correcta toma de decisiones, por consecuencia podemos minimizar efectos negativos y promover efectos favorables para las empresas. Estos métodos se pueden realizar de manera vertical y horizontal.

Haciendo referencia a Robles (2012, pág. 28), los métodos para el análisis financiero más importantes son:

\section{Porcientos integrales}

Razones Financieras

Modelo Dupont

Flujo de efectivo

Punto de equilirio

Ahora bien revisando a Gutiérrez (2006) muestra las técnicas de comparación en que se pueden clasificar las herramientas antes mencionadas.

\section{a. Método de análisis vertical o estático:}

Es una herramienta que se utiliza en el análisis financiero de una organización, es decir, la recopilación de información de los estados financieros, con esta información podemos interpretar los:

> Procedimiento de Porcientos integrales.

> Procedimiento de Razones Simples.

> Procedimiento del Balance Doble.

$>$ Procedimiento de Razones Estándar.

\section{b. Método de análisis horizontal o dinámico:}

Analiza los estados financieros, permitiendo observar las partidas a lo largo del tiempo, es de mayor utilidad para detectar fluctuaciones en la estructura de los estados financieros. de la misma empresa a fecha distinta o correspondiente a dos periodos o ejercicios.

> Procedimiento de aumentos y disminuciones.

\section{c. Método de análisis histórico:}

Es una herramienta financiera que analiza los estados financieros de una empresa a lo largo del tiempo, a fechas o periodos distintos.

Procedimiento de las tendencias, que para efecto de la comparación, estas se presentan en base a:

$>$ Serie de Cifras o Valores

$>$ Serie de Variaciones 
$>$ C. Serie de Índices.

\section{d. Método de análisis proyectado o estimado:}

Es una herramienta financiera donde analiza los estados financieros Pro-forma y presupuestos, se consideran los procedimientos presentados:

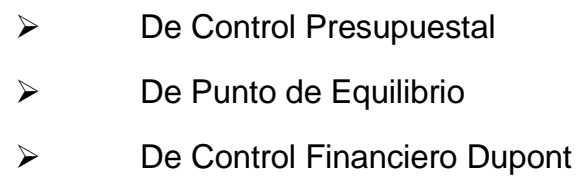

e. Método bursátil:

Herramienta financiera que examina los estados financieros de las compañías que cotizan acciones en la Bolsa de Valores.

Los métodos antes mencionados se encuentran descritos en la Figura 2.

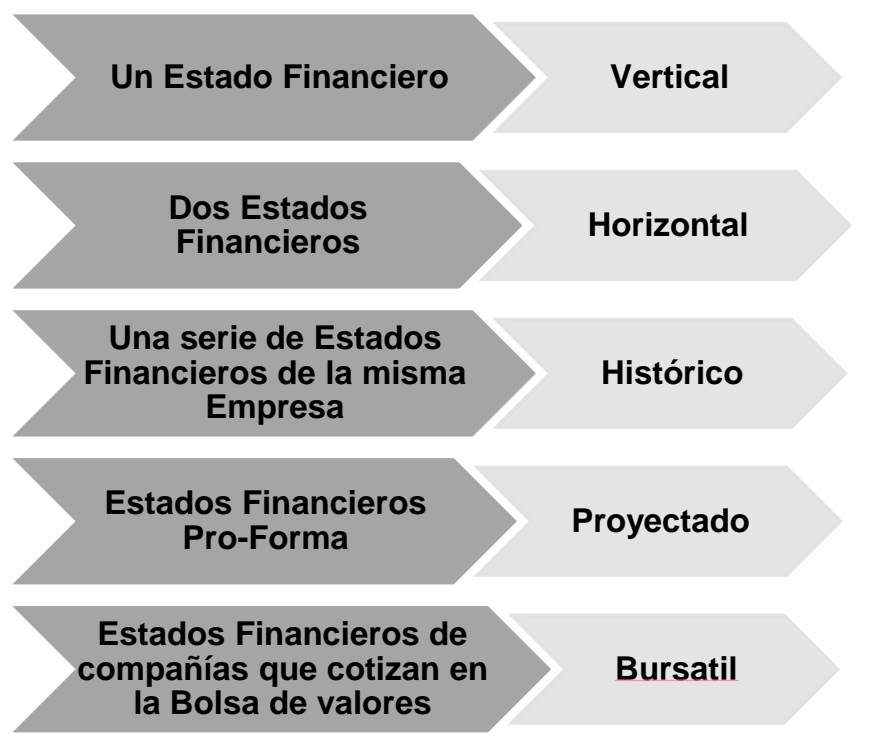

Figura 2. Fuente Propia obtenida de (LAE. GUTIERREZ REYNAGA, 2006, pág. 26)

\section{PORCIENTOS INTEGRALES}

De acuerdo con Gutiérrez (2006), el procedimiento de porcientos integrales muestra las cifras en porcentajes de un todo, es decir, expresa las partes integrales con el propósito de establecer la porción equivalente a cada una de las cantidades, se analizan los estados financieros a un mismo tiempo y correspondiente a un mismo periodo.
El estado de resultados representa como se obtuvieron, las ganancias o pérdidas desde el inicio de las ventas, si consideramos los porcientos integrales representa el porcentaje de participación de los costos y gastos, y entonces, las ventas estarían representando el $100 \%$ de un todo.

En el balance general se asigna el $100 \%$ a los activos totales y el $100 \%$ a la suma de pasivo más capital, de manera que, derivado de la partida de las cuentas anteriores, se va a estimar la parte integral equivalente a este.

A este procedimiento se le puede conocer como: Procedimiento de Porcientos Financieros; Procedimiento de Porcientos Comunes; Procedimiento de Reducción a Porcientos.

\section{RAZONES FINANCIERAS}

Es uno de las herramientas más usadas en el análisis de estados financieros, son comparables con las de la competitividad y obtienen el análisis y la abstracción del comportamiento de las empresas frente a sus rivales,

De acuerdo con (Robles Roman, Fundamentos de Administración Financiera, 2012), "Estas razones tienen como finalidad el estudio de cuatro indicadores fundamentales de las empresas: solvencia, actividad o productividad, endeudamiento y rentabilidad".

\section{MODELO DUPONT}

Tomando como base a Lawrence \& Chad (2012, pág. 68), es un modelo que reúne datos de los estados financieros, tomando como base el rendimiento sobre los activos (ROA) y los rendimientos sobre el capital (ROE)"

Haciendo referencia a Robles el Modelo Dupont "muestra la relación que existe entre el rendimiento sobre la inversión, la rotación de activos el margen de utilidad y el apalancamiento". (Robles Roman, Fundamentos de Administración Financiera, 2012). Donde la fórmula es una medida que indica el beneficio logrado por los inversionistas, tanto comunes como preferentes, de una empresa.

$$
\frac{\text { Poder ganancial }}{1-\frac{\text { Pasivos Totales }}{\text { Activos Totales }}}
$$


Para explicar mejor como funciona este modelo, presentamos el esquema mostrado en la Figura 3.

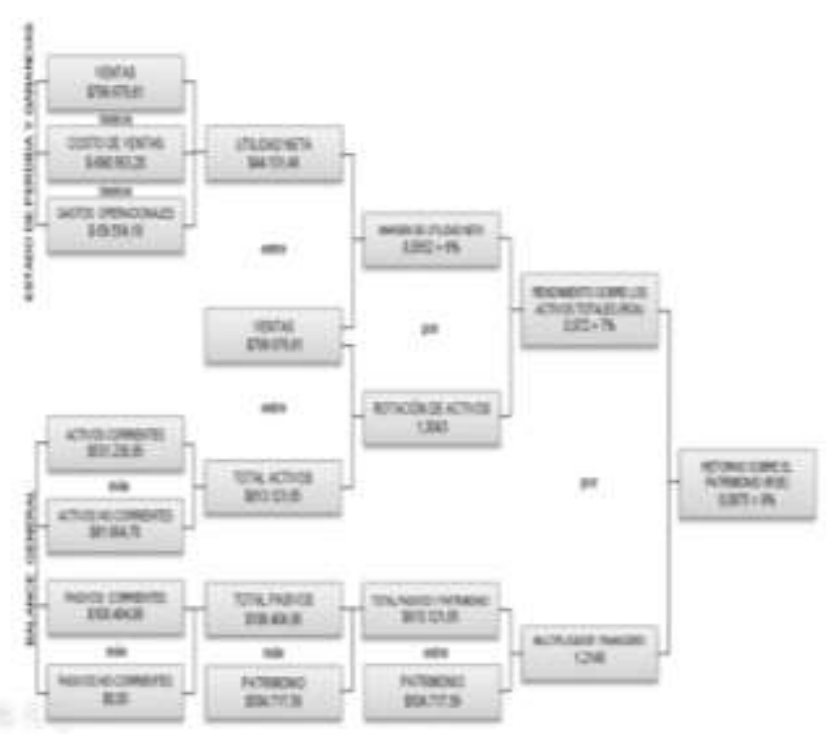

Figura 3. Ejemplo de Modelo Du Pont, Fuente obtenida de (Dra. Luzuriaga Granda \& Mgster. Ludeña Eras, 2018)

\section{FLUJO DE EFECTIVO}

El flujo de efectivo "Es un método que utilizan los administradores financieros para conocer si las empresas cuentan con los fondos necesarios para para poder realizar una operación" (Robles Roman, 2012) ,

Este método, también conocido como cash flow, permite realizar pronósticos, ayuda a gestión a la toma de decisiones, analiza las fluctuaciones en las entradas y salidas, colabora en el inspección de los ingresos con el propósito de lograr que las entidades sean rentables.

Para efectuar este flujo de efectivo se deben conocer todos los conceptos por los cuales se obtienen ingresos, correspondientes a las operaciones normales de las actividades propias del negocio o por financiamiento, también es necesario tener identificados los egresos en efectivo que se tienen que cubrir también de las operaciones normales o de financiamiento. Para ello se utiliza la estructura mostrada en la figura 4:

El flujo de efectivo se recomienda ser realizado a un plazo corto con el objeto de que sea más real, lo indicado es hacerlo a un año, desglosado mes a mes, además se le debe dar un seguimiento adecuado pues es un instrumento que apoya indiscutiblemente a la planeación.

\begin{tabular}{|c|c|c|c|}
\hline CONCEPTO & ENE & FEB & MAR \\
\hline $\begin{array}{l}\text { SALDO INICLAL } \\
\text { ENTRADAS: }\end{array}$ & 428,600 & 888,959 & 973,746 \\
\hline $\begin{array}{l}\text { VENTAS } \\
\text { RECUP, CTAS, POR COBRAR }\end{array}$ & $\begin{array}{r}4,962,687 \\
42,500 \\
\end{array}$ & $\begin{array}{r}4,553,995 \\
42,500 \\
\end{array}$ & $\begin{array}{r}3,911,766 \\
42,500 \\
\end{array}$ \\
\hline TOTAL DE ENTRADAS & $5,005,187$ & $4,596,495$ & $3,954,265$ \\
\hline $\begin{array}{l}\text { TOTAL DE DISPONIBLE } \\
\text { SALIDAS: (PAGOS) }\end{array}$ & $5,433,787$ & $5,485,455$ & $4,928,011$ \\
\hline COMPRA DE MATERIALES & $2,266,195$ & $1,672,795$ & $1,672,798$ \\
\hline SUELDOS Y SALARIOS & 315,728 & 315,728 & 315,728 \\
\hline GTOS INDIRECTOS & 789,320 & 789,320 & 789,320 \\
\hline GTOS DE VENTA & 247,184 & 278,082 & 185.388 \\
\hline GTOS DE ADMON & 369,768 & 415,989 & 277,326 \\
\hline GTOS FINANCIEROS & 10,300 & 11,588 & $7,72 t$ \\
\hline PAGO PROVEEDORES & 250,000 & 250,000 & 250,000 \\
\hline PAGO ACREEDORES & 143,333 & 143,333 & 143,334 \\
\hline PAGO DE IMPTOS (I.S.R., I.V.A & 153,000 & 634,875 & 587.846 \\
\hline TOTAL DE SALIDAS & $4,544,828$ & $4,511,709$ & $4,229,462$ \\
\hline SALDO EFECTIVO FIN DE MES & 888,959 & 973,746 & 698,648 \\
\hline
\end{tabular}

Figura 4. Ejemplo de Flujo de Efectivo, Fuente obtenida de (Robles Roman, 2012, pág. 38)

De acuerdo con (Ortega Castro, 2003, pág. 48), el procedimiento es el siguiente:

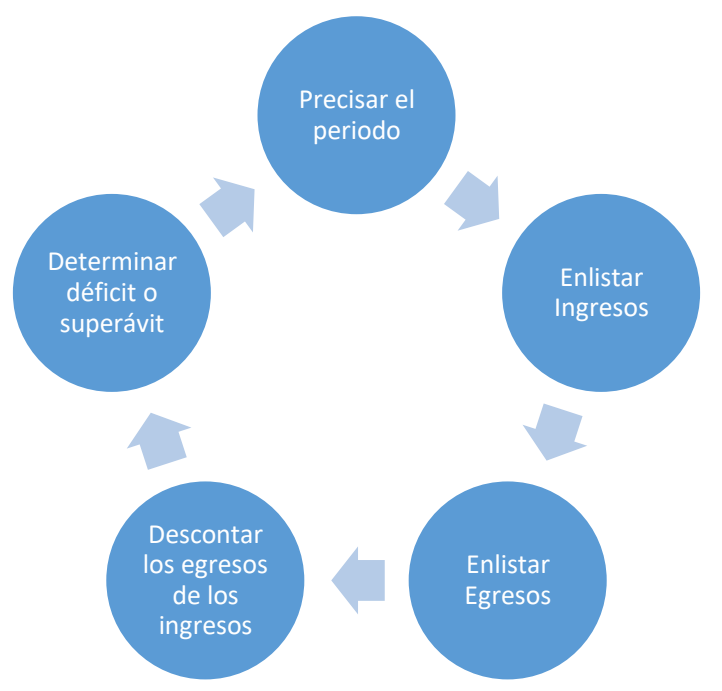

\section{PUNTO DE EQUILIBRIO}

Considerando a (Ortega Castro, 2003, pág. 48) "El índice del punto de equilibrio es un método que sirve como herramienta para realizar el presupuesto, que presenta de manera anticipada el nivel de ingresos que la empresa debe obtener para poder cubrir el total de gastos y costos, todo esto permite fijar los objetivos de ventas para lograr obtener las ganancias fijadas". 
El punto de equilibrio es más que el punto donde se juntan los ingresos con los egresos, y donde no se tienen pérdidas ni ganancias como comúnmente lo conocemos, ya que de acuerdo a (Robles Roman, 2012, pág. 40) existen dos.
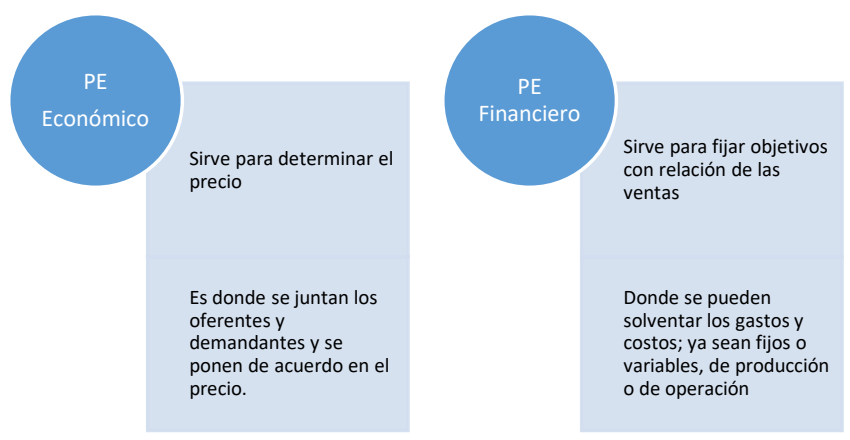

La fórmula para determinar el punto de equilibrio es la siguiente:

$$
\frac{\text { Costos Fijos }}{\text { Ventas - Costo Variable }}
$$

\section{Conclusiones}

Los estados financieros nos permiten conocer el comportamiento financiero que presentan las empresas, permitiendo evaluar la capacidad de la entidad para mantener y optimizar sus recursos, es decir, determinar la viabilidad del negocio en marcha. Mediante una representación bien estructurada, se debe presentar la situación financiera en la que se encuentran las organizaciones, el desempeño financiero y los flujos de efectivo de una empresa, para representar de manera fiable los escenarios futuros y los hechos económicos que las afectan representadas en transacciones, eventos, es decir y condiciones (contratos) de acuerdo con los criterios de reconocimiento CINIF y medición de las NIF/NIIF, cumpliendo con las normatividades en fechas determinadas como reflejo de su situación financiera y de los períodos comparados con años anteriores de su desempeño y resultado de sus flujos de efectivo.

\section{REFERENCIAS}

Deloitte. (12 de 09 de 2019). Deloitte. Obtenido de https://www2.deloitte.com/co/es/pages/ifrs_niif/n ormas-internacionales-de-la-informacionfinanciera-niif---ifrs-.html
Dra. Luzuriaga Granda, I., \& Mgster. Ludeña Eras, G. (2018). El Análisis Financiero como Estratégia de Gestión para Evaluar la Situación Financiera de las Empresas Comerciales. Revista Electrónica "Entrevista Académica", 25.

Felipe, P. C., Allan, . D., \& Antonio, C. M. (03 de 11 de 2019). Normas de Información Financiera (NIF) 2019. Obtenido de https://books.google.com.mx/books? isbn=60786 28259

HURTADO ROMERO, L. J. (2014). LA GERENCIA FINANCIERA EN LA TOMA DE DECISIONES. BOGOTÁ: “UNIVERSIDAD MILITAR NUEVA GRANADA, FACULTAD DE CIENCIAS ECONÓMICAS".

LAE. GUTIERREZ REYNAGA, C. G. (2006). FINANZAS I. Estado de México.

Lawrence J., G., \& Chad J., Z. (2012). PRINCIPIOS DE ADMINISTRACIÓN FINANCIERA. En G. Lawrence J., \& Z. Chad J., PRINCIPIOS DE ADMINISTRACIÓN FINANCIERA (pág. 720). México: PEARSON.

Ochoa Setzer, G. (2003). Administración financiera. En G. Ochoa Setzer, Administración financiera. McGraw-Hill.

Ortega Castro, A. (2003). Introducción a las Finanzas. En A. Ortega Castro, Introducción a las Finanzas. México: Mc. Graw Hill.

Robles Roman, C. L. (23 de 10 de 2012). Fundamentos de Administración Financiera. Obtenido de RED UNIVERCOM: https://www.upg.mx/wpcontent/uploads/2015/10/LIBRO-49Fundamentos-de-administracion-Financiera.pdf

Robles Roman, C. L. (23 de 10 de 2012). Fundamentos de Administración Financiera. Obtenido de RED UNIVERCOM: https://www.upg.mx/wpcontent/uploads/2015/10/LIBRO-49Fundamentos-de-administracion-Financiera.pdf 